\title{
FEATURE EXTRACTION METHOD FOR HIGH IMPEDANCE GROUND FAULT LOCALIZATION IN RADIAL POWER DISTRIBUTION NETWORKS
}

\author{
$\dagger \star$ Kåre Jean Jensen \\ ${ }^{\dagger}$ Research and Development Department \\ NESA A/S, Strandvejen 102 \\ DK-2900 Hellerup
}

\begin{abstract}
A new approach to the localization of high impedance ground faults in compensated radial power distribution networks is presented. The total size of such networks is often very large and a major part of the monitoring of these is carried out manually. The increasing complexity of industrial processes and communication systems lead to demands for improved monitoring of power distribution networks so that the quality of power delivery can be kept at a controlled level.

The ground fault localization method for each feeder in a network, is based on the centralized frequency broad band measurement of three phase voltages and currents. The method consists of a feature extractor, based on a grid description of the feeder by impulse responses, and a neural network for ground fault localization. The emphasis of this paper is the feature extractor, and the detection of the time instance of a ground fault.
\end{abstract}

\section{INTRODUCTION AND BACKGROUND}

Power delivery networks are split into two categories: the transmission network and the distribution network. The transmission network is connected to the power generation station and transports the energy over long distances to the local area. The distribution network is connected to the transmission network and distributes the energy to the customers. The object of this paper is a new method for detailed monitoring of the occurrence of ground faults (high impedance circuit from phase to earth) on the distribution network. Some networks tolerate a minor ground fault, but many ground faults develop into short circuits of phase to zero or phase to phase. The latter faults lead to malfunction of the feeder.

The transmission network, which can be considered as the backbone of the power delivery system, is monitored very detailed at a high level of reliability. This must be so, because faults in this part of the network will affect large parts of the distribution network, hence many customers.

\author{
* Department of Mathematical Modelling \\ Technical University of Denmark \\ DK-2800 Lyngby
}

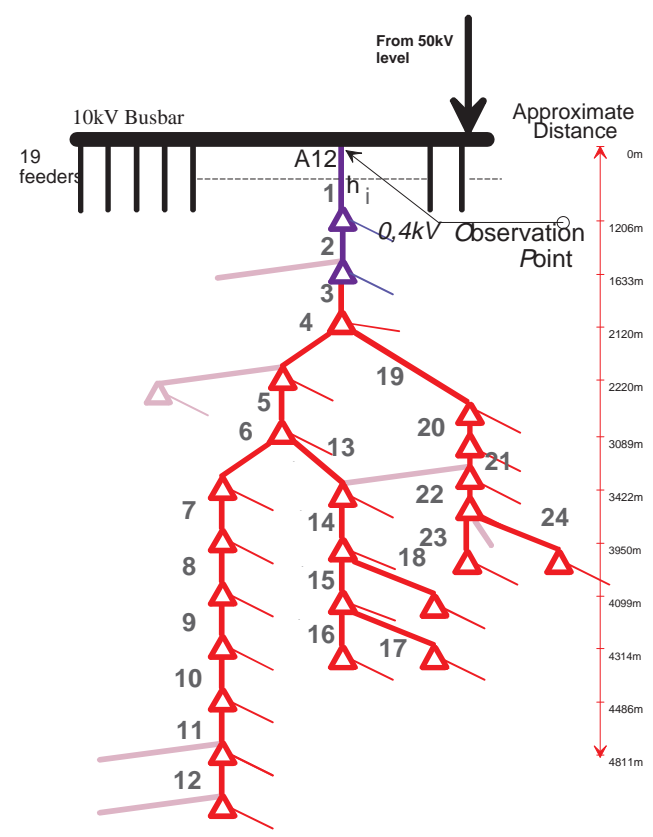

Figure 1: A12 at Glentegården, an example of a feeder.

The cost of this is a major amount of expensive auxiliary hardware and software.

In contrast to the transmission network monitoring, todays monitoring of the distribution network is very rudimentary. However, there is an increasing demand from customers to have a higher level of reliability in their electrical power supply. This originates in the steadily increasing complexity of industrial processes and communication systems.

The distribution network is much larger than the transmission network. E.g. the number of transformer stations on the distribution network is about two orders of magnitude larger than the number of stations on the transmission network. 


\subsection{The topology of the distribution network}

The topology of the distribution network, to which this monitoring method is targeted, consists of approx. 500000 customers at $0.4 \mathrm{kV}$ in NESA's ${ }^{1}$ area of operation. These customers are supplied from approx. 5800 secondary substations $(10 \mathrm{kV} / 0.4 \mathrm{kV}$ transformers, triangle symbols in Figure 1) and these are again supplied by approx. 600 feeders at $10 \mathrm{kV}$. The total length of NESA's distribution network is $4013 \mathrm{~km}$.

Because the physical extent and granularity of the distribution network is much larger than the transmission network, and because the impact of faults are more localized on the distribution network, it is necessary to devise new methods for system monitoring, which do not require substantial amounts of auxiliary hardware.

Most faults in the $10 \mathrm{kV}$ distribution network occur either in the cable/line or in cable joints. A large part of the faults on cables start as ground faults and develop into a short circuit. A ground fault is a (possibly) high impedance circuit from a phase to ground.

The distribution network considered is equipped with an arc suppression coil which reduces the effect of a ground fault. This means, that it might take a while, before the ground fault develops into a short circuit. It is therefore of interest to estimate the location of such faults, before they lead to interruption of the power supply.

\subsection{Existing localization methods}

Very few methods exist for localization of faults in power distribution networks. The existing methods all suffer from drawbacks in this context as they assumes conditions that are not present in a compensated branched network.

In [5] a method is described where a distance to the fault is computed by an iterative solution of equations which describe the steady state fault condition on the basis of the fundamental frequency component. In a branched network this gives multiple locations, so in order to estimate the location it is exploited that the fault interrupts the supply. This is not the case with high impedance ground faults in a compensated network, as the arc suppression coil minimizes the current in the ground fault.

In [1] the traveling time of the fault generated transient is used to estimate the fault location. This method will obviously have problems in a branched network as multiple reflections will be very difficult to track.

\section{OUTLINE OF THE GROUND FAULT DETECTION AND LOCALIZATION SYSTEM}

The detection and localization system [4] uses broad-band $(25 \mathrm{kHz})$ measurements of 3 -phase voltages and currents at

\footnotetext{
${ }^{1}$ NESA, see URL http: / / www . nesa.dk
}

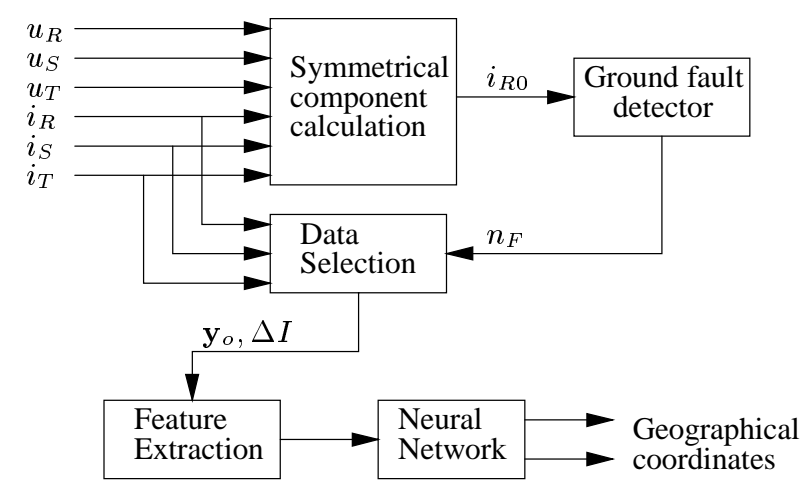

Figure 2: Ground fault detection system.

the supplying transformer station. The detection and localization of a ground fault then take place in three stages.

- The fundamental frequency voltage and current signals decomposed into the symmetrical components are used to detect the time instant of a ground fault event by thresholding the zero-system.

- The transient caused by the ground fault event and a grid model, based on FIR filters, of the feeder is used to make a feature extraction. This is the main topic of this paper.

- This feature extraction serves as input for a neural network which estimates the geographical location of the ground fault.

This procedure is illustrated in Figure 2 and will be outlined in the following.

The three phase voltages and currents of the $10 \mathrm{kV}$ feeder are measured at the supplying transformer station. These primary quantities are combined in the vector

$$
\mathbf{z}=\left[\begin{array}{llllll}
u_{R} & u_{S} & u_{T} & i_{R} & i_{S} & i_{T}
\end{array}\right]^{T}
$$

Under ideal, symmetrically loaded conditions the three voltages will differ only by a phase difference of $120^{\circ}$. The same, of course, applies to the currents. To make the symmetry explicit, $\mathbf{z}$ can be transformed to the corresponding symmetric components [3, Chapter 3.7] as, with reference to phase $R$, is defined by

$$
\mathbf{z}_{s, R}=\left[\begin{array}{cc}
\mathbf{S} & \mathbf{0} \\
\mathbf{0} & \mathbf{S}
\end{array}\right] \mathbf{z}
$$

where

$$
\mathbf{S}=\frac{1}{3}\left[\begin{array}{ccc}
1 & a & a^{2} \\
1 & a^{2} & a \\
1 & 1 & 1
\end{array}\right], \quad a=\mathrm{e}^{j \frac{2 \pi}{3}}
$$

Instead of the three phase voltages and currents, $\mathbf{z}_{s, R}$ contains a symmetrical, an inverse and a zero component for 
both the voltages and the currents. The symmetrical, inverse and zero component is the common part of the three phases that has a phase difference of $120^{\circ},-120^{\circ}$ and $0^{\circ}$ respectively. The zero component $i_{R 0}$ is only non-zero when a current returns through earth and can thus be used to detect the time instant $n_{F}$ of a ground fault by a threshold algorithm.

With the time index $n_{F}$ it is possible to select that part $\mathbf{y}_{o}$ of the relevant current signal containing the ground fault transient. A feature vector $\mathbf{f}$ and the increase $\Delta I$ in the phase current are extracted from $\mathbf{y}_{o}$. This is the main topic of this paper and is described in Section 3.

The feature vector $\mathbf{f}$ and the current increase $\Delta I$ can be used as input for a feed forward neural network [2]. Output of the neural network is two geographical coordinates pointing to the location where the ground fault occurred. By choosing this output as instead of an enumeration of cable segments e.g., it is ensured that the output is continuous around branch points of the network.

\section{FEATURE EXTRACTION FOR LOCALIZATION}

The basic idea is to represent the branched feeder by a set of impulse responses covering the feeder in a grid with equally spaced steps. In this way a large circuit simulation problem is converted to convolution of impulse responses. If $\mathbf{h}_{i}$ is the impulse response of the feeder from the $i$ 'th grid point to the observation point as shown in Figure 1 the feeder can be represented as a matrix

$$
\mathbf{H}=\left[\mathbf{h}_{0} \mathbf{h}_{1} \ldots \mathbf{h}_{N-1}\right]
$$

where $N$ is the number of grid points. Section 4.1 describes the generation of these impulse responses.

If $\mathbf{x}_{F}$ is the transient caused by the ground fault in grid point $F$, the measured transient at the observation point is given by the convolution

$$
\mathbf{y}_{o}=\mathbf{x}_{F} * \mathbf{h}_{F}
$$

where index $F$ is unknown.

An estimate of the transient at the fault point can be found by deconvolution as

$$
\hat{\mathbf{x}}_{i}=\mathbf{y}_{o} * \mathbf{h}_{i}^{-1}, \quad \mathbf{h}_{i} * \mathbf{h}_{i}^{-1}=\delta(n)
$$

In practice the deconvolution is carried out in the frequency domain.

By deconvolving a measured transient $\mathbf{y}_{o}$ to all positions in the impulse response grid, the corresponding feature vector is calculated as

$$
\mathbf{f}=\left[e_{i}^{2}\right]
$$

where

$$
e_{i}=\left\|\hat{\mathbf{x}}_{i-1}-\hat{\mathbf{x}}_{i}\right\|_{2}, \quad i=1, \ldots, N-1
$$

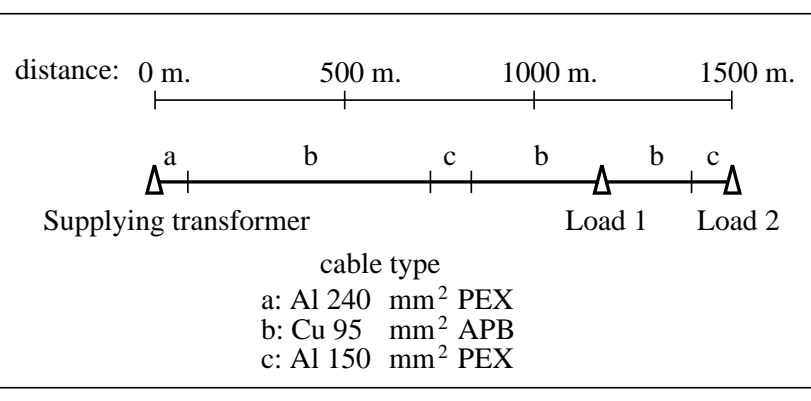

Figure 3: Feeder model and cable dimensions.

For a given measurement $\mathbf{y}_{o}$ the feature vector $\mathbf{f}$ is a function of the distance on the cable. Section 4.3 gives examples of this feature vector calculated on simulated data.

\section{SIMULATIONS}

The tool used for feeder modeling and ground fault simulation is the network simulation program EMTP 2 .

The model used for the simulations is a three phase model with mutually coupled $\Pi$-equivalents to model the cables. One $\Pi$-equivalent is used for every $10 \mathrm{~m}$ cable. The network being modeled is the first part of the feeder in Figure 1, and all parameters used are derived from this system and include cable lengths cable types and average loads at the distribution transformers. This information is normally accessible for the distribution utility. The model includes a total of $1550 \mathrm{~m}$. of cable, two loaded distribution transformers and three different types of cable distributed on 5 cable segments as shown in Figure 3. The arc suppression coil is not included in this model since the duration of the transients are shorter than $5 \mathrm{~ms}$ which is too fast for the coil to react.

\subsection{Impulse response model of feeder}

A central part of this localization method is that the complex network, made up of the feeder and containing mutually couplings, distributed loads and branches, is represented by a grid of impulse responses. The impulse response is calculated using EMTP by short circuiting the power generating voltage sources and connecting an impulse current source at the relevant grid point. The length of the impulse responses was chosen to be $50 \mathrm{~ms}$.

When the response at the observation point (see Figure 1) of a transient at a given location in the network needs to be found, a simple convolution gives the result. Compared to the processing time of the general simulation tools, this is a very efficient method.

\footnotetext{
${ }^{2}$ Electro Magnetic Transient Program is used to simulate transients on power delivery systems by solving partial differential equations in the time domain.
} 


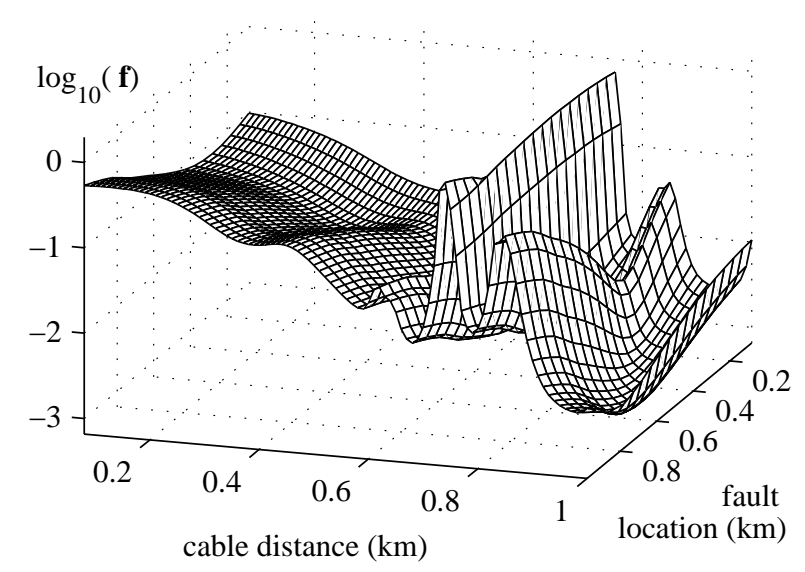

Figure 4: Feature vectors of varying fault locations for a ground fault impedance of $500 \Omega$.

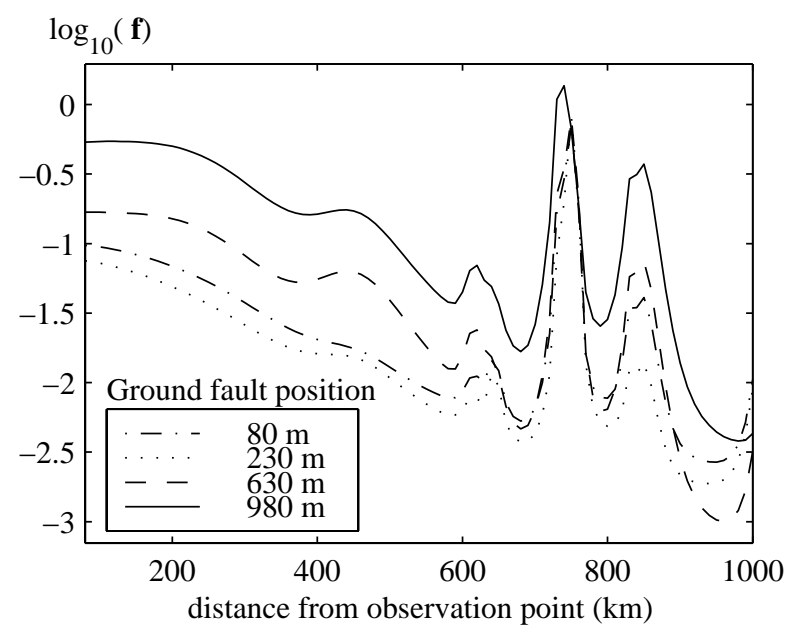

Figure 5: Feature vectors of three fault locations for a ground fault impedance of $500 \Omega$.

\subsection{Ground fault simulations}

The ground fault is simulated using a switch connecting a network node to ground through a resistance. A ground fault in a real network occurs when the voltage is around an absolute maximum so the switch is controlled accordingly during the EMTP simulation. The ground fault resistance used in this context is $500 \Omega$ which results in a current of $20 \mathrm{~A}$. This ground fault current is comparable in load to a large industrial machine.

\subsection{Feature extraction of ground fault simulations}

To show that this feature vector $\mathbf{f}$ in Eq. 7 is likely to be unique to a fault location consider Figure 4 and 5. It shows feature vectors $\mathbf{f}$ for the first $1000 \mathrm{~m}$. of the network in Fig- ure 3. Figure 4 shows feature vectors for simulated ground faults for every $50 \mathrm{~m}$. along the network. The question here is whether a neural network can map a given feature vector into the fault location position. Experiments with neural networks has not been made yet, but it seems likely that the surface in Figure 4 is sufficiently varying for a neural network to give a good performance. Figure 5 shows four of the feature vectors from Figure 4 for better comparison. It is seen that the different fault locations give feature vectors that are quite different and not only scaled versions of each other. An important point to note here is that the surface is smooth so a small change in the fault location will result in a correspondingly small change in the feature vector.

\section{CONCLUSION AND FURTHER WORK}

In this paper a feature extractor model for ground fault localization on a radial power distribution network is devised. The application of the model is exemplified on the first $1500 \mathrm{~m}$. of a specific distribution network feeder, which consists of 5 cable segments based on 3 different types of cable.

Further work will concentrate on validation of the localization method for a full scale testing facility with a power distribution radial of a total length of $6.5 \mathrm{~km}$.

It is evident that a single phase to ground fault will generate a transient in the two non-faulty phases as well as in the faulty phase. It is therefore likely that including feature information from the two non-faulty phases could be useful.

\section{REFERENCES}

[1] Z. Q. Bo, A. T. Johns, and R. K. Aggarwal. A Novel Fault Locator Based On The Detection of Fault Generated High Frequency Transients. Developments in Power System Protection, March 1997.

[2] S. Y. Kung. Digital Neural Networks. PTR PrentisHall, Inc., 1993.

[3] E. Lakervi and E. J. Holmes. Electricity Distribution Network Design. Peter Peregrinus Ltd., London, 1989.

[4] Steen M. Munk. Centralized Monitoring of $10 \mathrm{kV}$ Cable Based Radial Distribution Networks. PhD thesis, The Electronics Institute, Technical University of Denmark, August 1995.

[5] Jun Zhu, David L. Lubkeman, and Adly A. Girgis. Automated Fault Location and Diagnosis On Electric Power Distribution Feeders. IEEE Transactions on Power Delivery, 12:801-807, April 1997. 